\title{
2D Transformations Analyzed by Both Column Vector and Row Vector Synthesis
}

\author{
Shweta Chaku, Amrita Bhatnagar
}

\begin{abstract}
The 2D aspects of Computer Graphics such as vector primitives and $2 D$ transformations are important in creating $2 D$ content. The Transformation are the effective means of shifting or changing the dimensions and orientations of images in the most effective way. If we fail to transform the object in terms of displacement, enlargement, orientation, we may land up in creating something that is distorted and processessing a distorted object is not acceptable The usual practice of defining transformations is straight forward. The transformed object can be obtained by coupling original object with the transformation vectors .The main challenge is how to evaluate it. The usual practice is standard Column Vector form. The alternative Row Vector Form is also known approach but what matters is the sequence of operations that make these both approaches worth mentionin. While doing so our analysis on $2 D$ content keeps our knowledge flawless and takes it a step further as far as Image Processing is concerned. Such analytical study is very vital since most of the content created, acquired, reproduced, and visualized in $2 D$ needs to be mapped on to 3D.This paper describes the transformations(Translation,Scaling and Rotation) in the both Column and Row Vectar Approach. This paper aims in providing a clear sequence of calculations which differ in both approaches
\end{abstract}

Keywords: 2DTransformations, Homogeneous Coordinates, Rotation Scaling, Translation, Reflection

\section{INTRODUCTION}

Computer Graphics needs strong visualization of 2D and 3D objects. Consider a analogy of an architect who builds bridges. It becomes important for an architect to study bridges from different angles. i.e. Front View, Side View, Top View .All such Transformations require a complete analysis of displacement, zooming in zooming out and orientations. So if such images are converted into numbers, the numbers can be stored and manipulated for detailed analysis. This manipulation could be done using mathematical functions more precisely Matrix Operations. All of these transformations can be efficiently and succinctly handled using some simple matrix representations What makes it more overwhelming is the calculations behind all those transformation. My paper aims to compare the mathematical techniques laid down for such transformations and at the same time explores the distinct sequence of calculations by this comparison

\footnotetext{
Revised Manuscript Received on February 05, 2020.

* Correspondence Author

Shweta Chaku*, Assistant Professor, Department of Cloud Computing, OPJS University Churu Rajasthan, India.

Amrita Bhatnagar, Assistant Professor, Department of Digital Image Processing and Computer Organization, IPEC engineering college GZB.

(C) The Authors. Published by Blue Eyes Intelligence Engineering and Sciences Publication (BEIESP). This is an open access article under the CC BY-NC-ND license (http://creativecommons.org/licenses/by-nc-nd/4.0/)
}

\section{A. Classification of Transformations}

There are basically five transformations which are listed as below:
- Translation
- Scaling
- Rotation
- Reflection
- Shearing

\section{B. Objectives:}

The Objectives for laying this analytical methodology is important as far as transformations of 2D objects are concerned .They aim at

- Developing a clear semantics in laying down the mathematical formulae and

- Laying the proper sequencing of operation while performing the concatenation of the transformations

\section{Scope of the work:}

This work helps in analyzing the underlying formulae and comparison in two approaches towards transforming a 2D object

\section{METHODOLGY}

The methods in 2D transformations involve two different approaches:

- Column Vector Approach: In the Approach we multiply Column Vector Transformation matrix with the Original Object matrix to get the Transformed Object Matrix

- Row Vector Approach: In this Approach we multiply the Original Object Matrix with the Row Vector Transformation Matrix to get the Original Object Matrix

Analyzing the Column Vector Approach first followed by Row Vector Approach for 2D Transformations (will be considering only Translation ,Scaling and Rotation)

\section{A. TRANSLATION:}

It can be defined as displacement or shift of a 2D image by some vector along $\mathrm{x}$-axis and $\mathrm{y}$-axis 


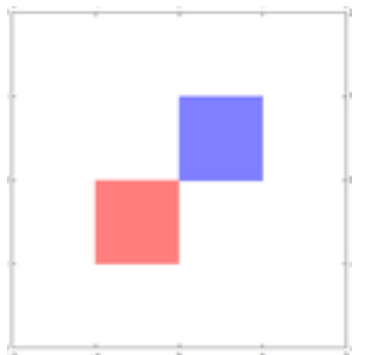

Fig[1]

We take a coordinate point as a starting point. What we need to show is the displacement of a original coordinate point to the translated coordinate point. Mathematically it can be formulated as shift vector along $\mathrm{x}$-axis and shift vector along $\mathrm{y}$-axis thus transforming the point from $\mathrm{P} 1$ to $\mathrm{P} 2$.Thus translation is defined by adding a shift vector in the $\mathrm{x}$ and $\mathrm{y}$ direction. These shift vectors can be termed as tx and ty(They are also called the translation vectors):Mathematically

$\mathrm{x}_{2}=\mathrm{x}_{1}+\mathrm{tx} ; \mathrm{y}_{2}=\mathrm{y}_{1}+\mathrm{ty}$ (Translation is Additive)

$$
\left[\begin{array}{l}
x_{2} \\
y_{2}
\end{array}\right]=\left[\begin{array}{l}
x_{1} \\
y_{1}
\end{array}\right]+\left[\begin{array}{l}
t_{x} \\
t_{y}
\end{array}\right]
$$

Other transformation matrices like Rotation ,Reflection )take different form so to get all matrices in a common form we introduce a homogenous system which means adding a dummy variable in $2 \mathrm{X} 2$ matrix to make it $3 \mathrm{X} 3$ matrix

\section{ORIGINAL APPROACH: TRANSLATION COLUMN VECTOR FORM}

IN

$$
\left[\begin{array}{c}
x_{2} \\
y_{2} \\
1
\end{array}\right]=\left[\begin{array}{ccc}
1 & 0 & t_{x} \\
0 & 1 & t_{y} \\
0 & 0 & 1
\end{array}\right] \cdot\left[\begin{array}{c}
x_{1} \\
y_{1} \\
1
\end{array}\right]
$$

Translated point with a column vector can be calculated by multiplying the translation matrix with the original coordinate.

\section{Thus $\mathbf{P} 2=\mathbf{T} \cdot \mathbf{P 1}$}

\section{ALTERNATIVEAPPROACH: TRANSLATION IN ROW VECTOR FORM}

The Row Vector form takes the translation vectors in the third row and the sequence of operation to get the transformed point take the reverse order. Original Point coordinate has to be multiplied with translation matrix to get the transformed point

$$
\left[\begin{array}{lll}
x^{\prime} & y^{\prime} & 1
\end{array}\right]=\left[\begin{array}{lll}
x & y & 1
\end{array}\right] \cdot\left[\begin{array}{ccc}
1 & 0 & 0 \\
0 & 1 & 0 \\
t_{x} & t_{x} & 1
\end{array}\right]
$$

\section{Alternatively write $\mathbf{P} 2=P 1 \cdot T$}

B. SCALING:

Scaling is defined as the process of changing the size of an object in the $\mathrm{x}$ and $\mathrm{y}$ direction.(Resizing can change shape also).Scaling with respect to origin can be done by multiplying the coordinate valuesP1 $(\mathrm{x}, \mathrm{y})$ of each vertex of a object by scaling factors sx,sy respectively to produce coordinate $\mathrm{P} 2\left(\mathrm{x}^{\prime}, \mathrm{y}^{\prime}\right)$. In this process, we can either enlarge or shrink the object.Such a scaling is called Pure scaling.Value of scaling factors can effect our object in a greater way for e.g; if $\mathrm{sx}=\mathrm{sy}>1$,it means Uniform Extention.If $s x=s y<1$ means Uniform Reduction and if $s x$ is not equal to sy, it means Shape Distortion

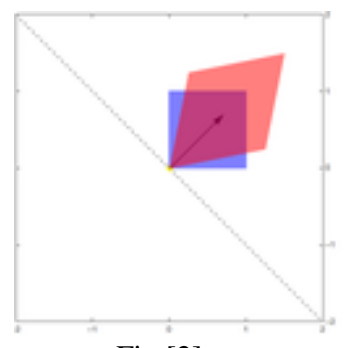

Fig [2]

The relation is: $\mathrm{x}_{2}=\mathrm{x}_{1} \cdot \mathrm{sx}$ and $\mathrm{y}_{2}=\mathrm{y}_{1} \cdot \operatorname{sy}$ (Scaling is Multiplicative)

\section{ORIGINAL APPROACH: SCALING IN COLUMN} VECTOR FORM

$$
\left[\begin{array}{c}
x_{2} \\
y_{2} \\
1
\end{array}\right]=\left[\begin{array}{ccc}
s_{x} & 0 & 0 \\
0 & s_{y} & 0 \\
0 & 0 & 1
\end{array}\right] \cdot\left[\begin{array}{c}
x_{1} \\
y_{1} \\
1
\end{array}\right]
$$

A Transformed point with a column vector can be calculated by multiplying the scaling matrix with the original coordinate.

\section{Thus $\quad \mathbf{P} 2=\mathbf{T} \cdot \mathbf{P} 1$}

\section{ALTERNATIVE APPROACH: SCALING IN ROW VECTOR FORM}

The Scaling matrix takes the same form both in Column Vector Approach as well as in Row Vector Approach. We can confirm it by rewriting the scaling matrix by arranging the elements in column as row. Importantly transformed point with a column vector can be calculated by multiplying the original coordinate with the scaling matrix.

$$
\left[\begin{array}{lll}
x^{\prime} & y^{\prime} & 1
\end{array}\right]=\left[\begin{array}{lll}
x & y & 1
\end{array}\right] \cdot\left[\begin{array}{ccc}
s_{x} & 0 & 0 \\
0 & s_{y} & 0 \\
0 & 0 & 1
\end{array}\right]
$$

\section{Thus Alternatively $\mathbf{P 2}=\mathbf{P} 1 . \mathbf{T}$}

\section{ROTATION:}

Rotation is expressed relative to origin. We can rotate a $2 \mathrm{D}$ object by any angle.

We get

$$
\begin{aligned}
& \mathrm{P}_{1}=\left(\mathrm{x}_{1}, \mathrm{y}_{1}\right)=(\mathrm{r} \cdot \cos (\mathrm{a}), \mathrm{r} \cdot \sin (\mathrm{a})) \\
& \mathrm{P}_{2}=\left(\mathrm{x}_{2}, \mathrm{y}_{2}\right)=(\mathrm{r} \cdot \cos (\mathrm{a}+\mathrm{b}), \mathrm{r} \cdot \sin (\mathrm{a}+\mathrm{b}))
\end{aligned}
$$

$$
\begin{aligned}
P_{1}= & ((r \cdot \cos (a), r \cdot \sin (a) \\
P_{2}= & (r \cdot \cos (a) \cdot \cos (b)-r \cdot \sin (a) \cdot \sin (b), \\
r & r \cdot \cos (a) \cdot \sin (b)+r \cdot \sin (a) \cdot \cos (b))
\end{aligned}
$$

We insert:

$$
\begin{aligned}
\mathrm{x}_{1} & =\mathrm{r} \cdot \cos (\mathrm{a}) \\
\mathrm{y}_{1} & =\mathrm{r} \cdot \sin (\mathrm{a})
\end{aligned}
$$

in P2's coordinates 
$\mathrm{P}_{2}=\left(\mathrm{x}_{2}, \mathrm{y}_{2}\right)=(\mathrm{x} 1 \cdot \cos (\mathrm{b})-\mathrm{y} 1 \cdot \sin (\mathrm{b}), \mathrm{x} 1 \cdot \sin (\mathrm{b})+\mathrm{y} 1 \cdot \cos (\mathrm{b}))$ and we have expressed P2's coordinates with P1's coordinates and the rotation angle $\mathrm{v}$.

$$
\begin{aligned}
& \mathrm{x}_{2}=\mathrm{x}_{1} \cdot \cos (\mathrm{v})-\mathrm{y}_{1} \cdot \sin (\mathrm{v}) \\
& \mathrm{y}_{2}=\mathrm{x}_{1} \cdot \sin (\mathrm{v})+\mathrm{y}_{1} \cdot \cos (\mathrm{v})
\end{aligned}
$$

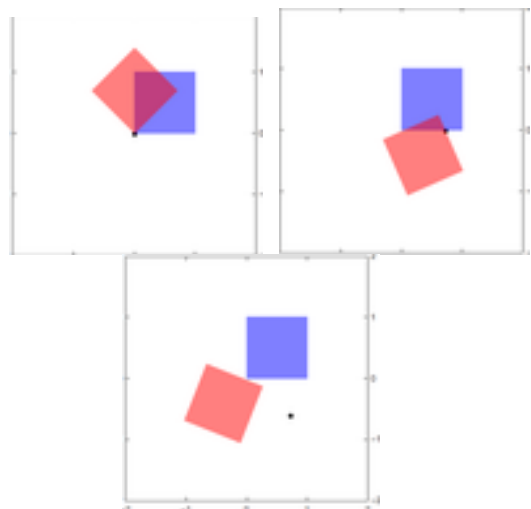

Fig [3]

$$
\left[\begin{array}{c}
x_{2} \\
y_{2} \\
1
\end{array}\right]=\left[\begin{array}{clc}
\cos (v) & -\sin (v) & 0 \\
\sin (v) & \cos (v) & 0 \\
0 & 0 & 1
\end{array}\right] \cdot\left[\begin{array}{c}
x_{1} \\
y_{1} \\
1
\end{array}\right]
$$

Thus $\quad \mathbf{P 2}=\mathbf{M} \cdot \mathbf{P} 1$

\section{ALTERNATIVE APPROACH:ROTATION IN ROW VECTOR FORM}

$$
\left[\begin{array}{lll}
x^{\prime} & y & 1
\end{array}\right]=\left[\begin{array}{lll}
x & y & 1
\end{array}\right] \cdot\left[\begin{array}{cll}
\cos (v) & \sin (v) & 0 \\
-\sin (v) & \cos (v) & 0 \\
0 & 0 & 1
\end{array}\right]
$$

\section{Alternatively $\quad \mathbf{P} 2=\mathbf{P 1}$.M}

\section{RESULT AND DISCUSSION}

We can set up a matrix for any number or sequence of transformations as a composite transformation matrix by calculating the matrix product for the individual transformations. It is often referred as concatenation or composition of matrices. In concatenation of transformations, the sequence of the transformations play a vital role. This sequence is written from Right to Left(R$>\mathrm{L}$ ) in column Approach of synthesis.But in Row Approach the sequence takes the order from Left to Right(L->R). $[\mathrm{T} 1] *[\mathrm{~T} 2]$ is not equal to the $[\mathrm{T} 2] *[\mathrm{~T} 1]$.

\section{CONCLUSION}

It can be concluded that if as an graphics analyst we don't draw the clear comparisons between the both approaches we might compromise on the issues of having simple ,consistent matrix notation using Homogenous Coordinate System and finally land up in misinterpretation of window modeling. Thus it is important to have a clear understanding between the sequencing of operations in both the approaches .Concatenation of Transformations multiplied with the Original Object in Column Vector Approach will equate same with Row Vector Approach only if Original Object is multiplied with Concatenation of Transformations

\section{REFERENCES}

1. Ms.A.J.Rajeswari Joe, Dr.N.Rama, "Scaling Transform Methods Advanced Computing : An International Journal ( ACIJ ), Vol.4, No.2, March 2013.

2. Computer Graphics by Hearn and M. Baker, 2 Edition

3. Schaum's outlines Computer Graphics Second Edition

4. https://demonstrations.wolfram.com/Understanding2DTranslation(URL for Fig[1],Fig[2],Fig[3])

5. Manoj Kumar Srivastav Champdani Adarsh Sharmik Vidyamandir, 3, R. B. S. Road, Champdani, "transformation of an Object in Computer Graphics: A Case Study of Mathematical Matrix Theory "(Elixir International

6. Srivastav, M. K. (2016). Transformation of an Object in Computer Graphics: A Case Study of Mathematical Matrix Theory. Elixir Comp. Engg. 100, 43396-43399

7. Procedural Elements for Computer Graphics by David F. Rogers Second Edition

8. Kartik, Seth Jai Parkash Mukand Lal. "A Descriptive Study of 2D Graphics Transformations", ISSN(Online): 2320-9801ISSN (Print): 2320-9798 International Journal of Innovative Research in Computer and Communication Engineering

\section{AUTHOR PROFILE}

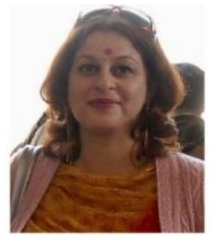

Shweta chaku, is a Assistant Professor in IPEC Engineering College persuing Phd from OPJS University Churu Rajasthan, Her research area includes Cloud Computing.She obtained MTech from, Karnataka University in Information Technology, BTech in Information Technology from BVPCOE (Bharti Vidyapeeth College of Engineering Pune) and D.E.E (Three years Diploma in Electrical Engineering) from Bombay Technical Board .Her interest area includes Computer Graphics,DataBase Management System ,Software Engineering,Principles of Programming Language and many more. She has published papers in reputed Journals .

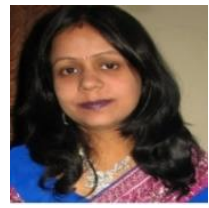

Amrita Bhatnagar, is a Assistant Professor in IPEC engineering college GZB.She is BE from SRMSCET Bareilly and Mtech from BIT Mesra Rachi.Her research area include Digital Image Processing and computer Organization.She has published papers in reputed Journals. 